\title{
Incidence of and Reasons and Determinants Associated with Retransitioning from Biosimilar Etanercept to Originator Etanercept
}

\author{
Rosanne W. Meijboom ${ }^{1,2} \cdot$ Helga Gardarsdottir ${ }^{2,3,4} \cdot$ Matthijs L. Becker ${ }^{1,5} \cdot$ Saskia ten Wolde ${ }^{6}$. Toine C. G. Egberts ${ }^{2,3}$. \\ Thijs J. Giezen ${ }^{1,2,5}$
}

Accepted: 28 September 2021 / Published online: 26 October 2021

(c) The Author(s) 2021

\begin{abstract}
Background Patients in clinical practice are transitioned from originator etanercept (OR-ETA) to biosimilar etanercept (BSETA), but some subsequently retransition. Insights into the incidence of and reasons for retransitioning and the characteristics of these patients could help clinicians successfully introduce biosimilars.

Objective Our objective was to assess the incidence of and reasons for retransitioning from BS-ETA to OR-ETA in patients with a rheumatic disease (RD) and to explore the determinants thereof.

Methods This cohort study included all patients with RD who had transitioned from OR-ETA to BS-ETA in a large hospital in the Netherlands in 2016. The incidence of retransitioning to OR-ETA and the 1-year persistence with BS-ETA were assessed using the Kaplan-Meier estimator. Reasons for retransitioning were classified as related to (1) efficacy, (2) adverse events, (3) the administration device, and (4) other. Determinants for retransitioning, including baseline and treatment characteristics, were assessed in a nested case-control study using conditional logistic regression.

Results We included 342 patients (median age 57.8 years; 53.5\% females). At 1 year after transitioning, 9.4\% of patients had retransitioned to OR-ETA and 69.7\% were persistent with BS-ETA. At the end of follow-up (median 4.4 years), 47 patients (13.7\%) had retransitioned to OR-ETA. The median time until retransitioning was 0.55 years (interquartile range $0.2-1.3)$. Most patients $(n=34$ [72.3\%]) retransitioned because of a (perceived) loss of effect, followed by adverse events (23.4\%). In total $3.8 \%$ of patients switched to another biological treatment or a Janus kinase inhibitor; $17.1 \%$ of patients discontinued BS-ETA without retransitioning or switching within the first year. Univariate determinants for retransitioning included initiating corticosteroids or intensifying immunomodulator treatment (odds ratio [OR] 2.37; 95\% confidence interval [CI] 1.03-5.45) and the number of visits to the rheumatology department (OR 2.06; 95\% CI 1.55-2.74). In the multivariate analysis, only the number of visits to the rheumatology department remained significantly associated with retransitioning (OR 2.19; 95\% CI 1.60-3.01).
\end{abstract}

Conclusion When introducing a biosimilar in clinical care, clinicians should anticipate that one in seven patients will retransition to the originator. A (perceived) loss of effect was the most frequently reported reason for retransitioning. Patients who visited the rheumatology department more frequently had an increased risk of retransitioning, which is likely to be related to patients reporting a loss of effect and to adverse events resulting in more visits to the rheumatology department.

Thijs J. Giezen

tgiezen@sahz.nl

1 Pharmacy Foundation of Haarlem Hospitals, Haarlem, The Netherlands

2 Division of Pharmacoepidemiology and Clinical Pharmacology, Utrecht Institute for Pharmaceutical Sciences, Utrecht University, Utrecht, The Netherlands

3 Department of Clinical Pharmacy, University Medical Centre Utrecht, Utrecht, The Netherlands
4 Department of Pharmaceutical Sciences, University of Iceland, Reykjavík, Iceland

5 Department of Clinical Pharmacy, Spaarne Gasthuis, Haarlem and Hoofddorp, The Netherlands

6 Department of Rheumatology, Spaarne Gasthuis, Haarlem and Hoofddorp, The Netherlands 


\section{Key Points}

After patients transition from an originator to a biosimilar, approximately one in seven will retransition to the originator.

The most frequently reported reason for retransitioning was a (perceived) loss of effect, followed by adverse events.

An increased number of visits to the rheumatology department was related to an increased risk of retransitioning, which probably reflects patients experiencing loss of effect and/or adverse events.

\section{Introduction}

Several tumor necrosis factor (TNF)- $\alpha$ inhibitor biosimilars have been available in Europe and the USA for several years. The market exclusivity right for originator etanercept expired in Europe in January 2016, and the first etanercept biosimilar was introduced in clinical practice soon thereafter. A biosimilar is defined as a "biological medicinal product that contains a version of the active substance of an already authorized biological medicinal product (originator)" [1]. The similarity of the biosimilar etanercept to the originator was demonstrated by an extensive comparability exercise comparing physiochemical properties, biological activity, immunochemical properties, and in vivo pharmacological properties [2]. Finally, efficacy and safety were studied and similarity confirmed in three premarketing clinical studies, which randomly assigned TNF $\alpha$ inhibitor-naïve patients with rheumatoid arthritis to originator etanercept or to the biosimilar [3-5].

The market entry of the etanercept biosimilar, as with all biosimilars, led to competition, reduced prices, and reduced financial burdens for healthcare budgets. Therefore, many patients in clinical practice are currently transitioned from originator etanercept to the biosimilar. In a phase III randomized controlled trial (RCT), patients were blindly transitioned from originator etanercept to a biosimilar, and the results confirmed that transitioning to the etanercept biosimilar did not affect efficacy, safety, or immunogenicity [6].

However, the results of RCTs in which patients transitioned from originator etanercept to the biosimilar have not been reflected in observational studies. Patients who transitioned from originator etanercept to the biosimilar identified from the DANBIO registry in Denmark remained stable in their disease activity but had a significantly lower 1-year persistence of $82 \%$ (95\% confidence interval [CI] 79-83) compared with $88 \%$ (95\% CI 87-90) in the historic cohort of originator etanercept users [7]. Similar results were reported in the Dutch BIOSPAN study, where patients who transitioned from originator etanercept to the biosimilar had a higher relative risk (hazard ratio [HR] 1.57; 95\% CI 1.05-2.36) of treatment discontinuation. Patients who transitioned also experienced more subjective adverse events than did users of the originator (84 vs. 40\%) [8].

Moreover, in the aforementioned observational studies and others, $2.7-17.2 \%$ of patients who transitioned from originator etanercept to the etanercept biosimilar retransitioned to originator etanercept within 6-12 months [7-10]. The most important reasons for retransitioning were adverse events caused by the etanercept biosimilar, including subjective adverse events such as arthralgia and fatigue, or (perceived) loss of effect. According to the authors of these studies, the higher rate of subjective adverse events after transitioning to the etanercept biosimilar could have been caused by the nocebo effect (where negative perceptions of transitioning to a biosimilar result in unwanted effects [9]); however, this has not been explicitly studied.

Current studies have not provided insight into which types of patients are more likely to retransition to originator etanercept. An important consideration for clinicians is whether a successful transition from the originator to the biosimilar can be achieved with a limited burden on the patient. Insights into the incidence of and reasons for retransitioning and the characteristics of patients who are most likely to retransition could help clinicians ensure the successful introduction of biosimilars.

The aims of this study were to assess the incidence of and reasons for retransitioning from biosimilar etanercept to originator etanercept in patients with a rheumatic disease (RD) and to explore the determinants of retransitioning.

\section{Methods}

\subsection{Setting and Study Population}

This cohort study was conducted at the Spaarne Gasthuis, a large teaching hospital in Haarlem and Hoofddorp, the Netherlands. In line with current Dutch reimbursement regulations, all biologicals used for the treatment of RDs in the outpatient setting have been exclusively dispensed by the outpatient pharmacy of the treating hospital since 2012 [11].

Patients treated with etanercept for RD and who transitioned from originator etanercept to biosimilar etanercept between June 2016 and December 2016 were included. The date at which a patient first received biosimilar etanercept was assigned as that patient's index date. Patients were 
followed from the index date until retransitioning to originator etanercept, switching to another biological or Janus kinase (JAK) inhibitor, discontinuing biological treatment, being lost to follow-up, or death or reaching the end of data collection (18 April 2021), whichever came first.

In the Netherlands, the decision to transition patients to a biosimilar is made by individual hospitals; transitioning is directed by treating physicians and hospital pharmacists. All patients in this study received a letter to inform them about the introduction of biosimilar etanercept and an additional information package about the biosimilar. Patients received their usual care during the transition. However, patients had the option of consulting their rheumatology nurse or rheumatologist if they had questions or concerns about transitioning to biosimilar etanercept or if they required extra training by the rheumatology nurse on using the biosimilar etanercept autoinjector. The information and communication on transitioning was consistent throughout the whole period (June-December 2016).

\subsection{Retransitioning}

For each included patient, treatment episodes for the biosimilar etanercept were constructed. These episodes were defined as the time between the first dispensing of biosimilar etanercept until the end of the duration of the final dispensing within the treatment episode, calculated based on the number of syringes dispensed and the dosing frequency. The information required to construct the episodes (dose and dosing regimen, specialism of the prescriber, and dispensing date) was collected from CompuGroup Medical (Echt, the Netherlands), an outpatient pharmacy system. A maximum permissible gap of 90 days was allowed to elapse between the theoretical end date of a dispensing and the subsequent dispensing date. The 90-day limit was based on the 90-day standard dispensing period in the Netherlands, which is applicable to clinical practice at the Spaarne Gasthuis.

Retransitioning was defined as restarting originator etanercept within the maximum permissible gap of 90 days from the theoretical end date of biosimilar etanercept dispensing. The date of retransitioning was assigned as the patient's event date. The reason for retransitioning was extracted from the electronic patient dossier EPIC (Madison, WI, USA) and classified as related to (1) efficacy, (2) an adverse event, (3) the autoinjector through which the biosimilar was administered, or (4) other.

We also assessed persistence on biosimilar etanercept, the incidence of switching from biosimilar etanercept to another biological or JAK inhibitor, and the incidence of discontinuing etanercept treatment without switching. Persistence (continuous use) was assessed at 6 months, 1 year, and end of follow-up. Switching was defined as dispensing of another biological or JAK inhibitor (listed in the electronic supplementary material [ESM]-S1) registered for RD within the maximum permissible gap after the theoretical end date of the final dispensing of biosimilar etanercept. Discontinuing biosimilar etanercept without switching was defined as no dispensing of biosimilar etanercept within the maximum permissible gap without retransitioning or switching.

\subsection{Patient- and Treatment-Related Characteristics Associated with Retransitioning}

To explore the patient- and treatment-related characteristics associated with retransitioning, we performed a nested case-control study. Cases were defined as patients who retransitioned from biosimilar etanercept to originator etanercept. Up to four controls were randomly selected for each case using incidence density sampling. Cases and controls were matched by index date (index date between June and August 2016 or between September and December 2016) to correct for potential seasonal influences during transitioning.

The following characteristics were explored: age at index date (continuous, years); sex (male or female); biosimilar etanercept dosing interval at index date (7 days [12] or more than 7 days); use of other biologicals registered for $\mathrm{RD}$ prior to originator etanercept (yes or no); duration of originator etanercept treatment before index date (longer or shorter than the median duration of originator etanercept treatment); initiation of corticosteroids or intensification of immunomodulator treatment in the 60-day period before the event (yes or no); hospitalizations, defined as having been hospitalized (yes or no) within 6 months before the event, included as a representation of the general health condition of the patient; and number of outpatient visits to the rheumatology department, defined as the sum of the number of outpatient visits and phone consultations with the rheumatology department in the 60-day period before the event (continuous).

The included immunomodulators and corticosteroids are listed in ESM-S2.

\subsection{Data Analysis}

Descriptive statistics were used to present the characteristics of the patients and the reasons for retransitioning. Time on biosimilar etanercept was presented with a Kaplan-Meier curve. The cumulative incidence of retransitioning, switching, or discontinuing was presented in cumulative incidence curves. Patient and treatment characteristics associated with retransitioning were explored using conditional logistic regression and expressed as odds ratios (ORs) with corresponding 95\% CIs. All characteristics were included in the multivariate model. 
Data were analyzed using R version 3.6.1.

\section{Results}

In total, 342 patients transitioned to biosimilar etanercept during the study period and were thus included in our cohort. An overview of the included patients is presented in Fig. 1. The median age of the patients was 57.8 years, and $53.5 \%$ were female. For a majority of patients $(93.0 \%)$, originator etanercept was the first biological treatment, and the median duration of originator etanercept treatment prior to the index date was 4.3 years. At the index date, the median dosing interval was 7 days, which is in line with the approved dosing interval (Table 1).

In total, $9.4 \%$ of the patients had retransitioned to originator etanercept 1 year after the index date and 47 patients (13.7\%) had retransitioned at the end of follow-up (median 4.4 years). The median time until retransitioning was 0.55 years (interquartile range [IQR] 0.2-1.3) (Fig. 2). (Perceived) loss of effect after the introduction of the biosimilar was the most frequently reported reason for retransitioning $(n=34$ [72.3\%]). Patients reported, among other symptoms, an increase in pain, swelling of joints, and (morning) stiffness, and 11 (23.4\%) patients reported one or more adverse event resulting in retransitioning to originator etanercept. The type of adverse events reported varied, but the most frequently reported was an itching skin reaction (four patients). No patients retransitioned because of the autoinjector through which biosimilar etanercept was administered. Two patients $(4.2 \%)$ retransitioned for other reasons.

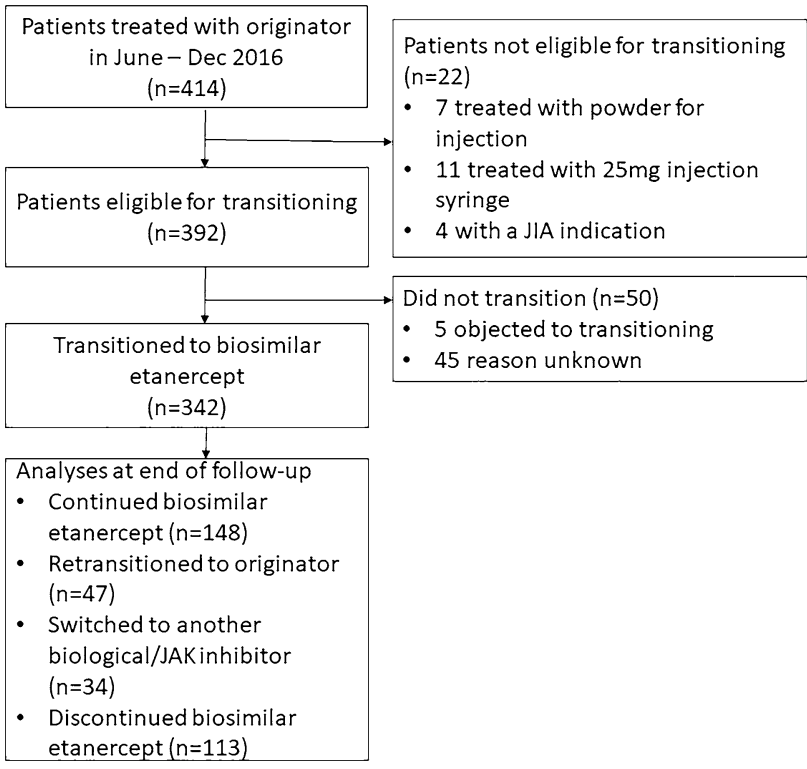

Fig. 1 Flow chart of included patients. JAK Janus kinase, JIA juvenile idiopathic arthritis
Table 1 Baseline characteristics of the study cohort $(n=342)$

\begin{tabular}{ll}
\hline Patient and treatment characteristics & $n=342$ \\
\hline Females & $183(53.5)$ \\
Age at index date, years & $57.8(47.6-67.7)$ \\
Dosing interval at index date, days & $7(7-10)$ \\
Number of biologicals used before index date & \\
0 & $318(93.0)$ \\
1 & $16(4.7)$ \\
2 & $7(2.0)$ \\
$>2$ & $1(0.3)$ \\
Duration of originator etanercept treatment, years & $4.3(2.8-4.6)^{\mathrm{a}}$ \\
Follow-up time from index date, years & $4.4(4.1-4.6)$ \\
\hline
\end{tabular}

Data presented as $n(\%)$ or median (interquartile range) unless otherwise specified

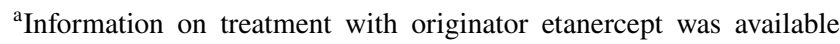
from January 2012; patients could have been treated for a longer period

Aside from retransitioning, the persistence with biosimilar etanercept was $82.4 \%$ at 6 months and $69.7 \%$ at 1 year. In total, $3.8 \%$ of patients switched to another biological treatment or a JAK inhibitor; $17.1 \%$ of patients discontinued biosimilar etanercept without retransitioning or switching within the first year.

Patients who retransitioned remained treated with the originator for a median of 2.0 years (IQR 0.8-4.0). Eight of the 47 retransitioned patients (17.0\%) switched to another biological within a median of 1.0 years (IQR $0.5-1.5$ ) after retransitioning. These patients switched to adalimumab ( $n$ $=2)$, baricitinib $(n=1)$, secukinumab $(n=2)$, rituximab ( $n$ $=1)$, tocilizumab $(n=1)$, or ustekinumab $(n=1)$.

As depicted in Fig. 2, at the end of follow-up, 33.0\% of the patients $(n=113)$ had discontinued biosimilar etanercept treatment without retransitioning or switching. Of these

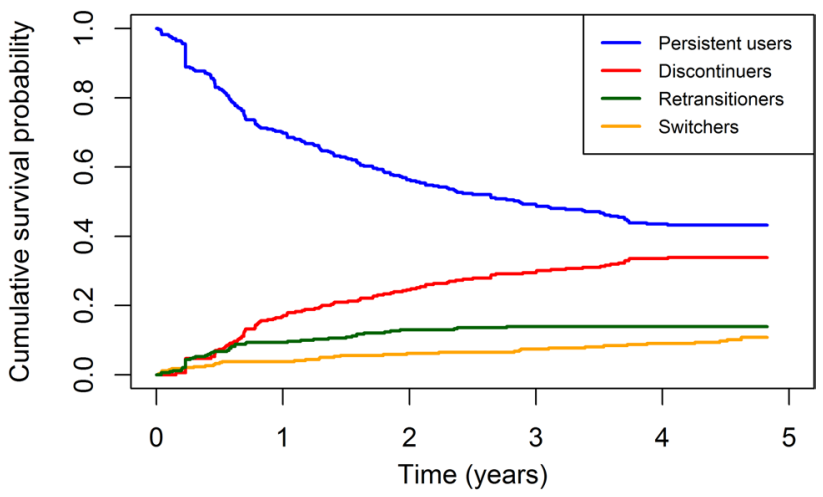

Fig. 2 Kaplan-Meier curve of time on biosimilar etanercept and time until retransitioning; time until switch to another biological and time until discontinuation of biological treatment 
discontinued patients, 78.8\% $(n=89)$ restarted biosimilar etanercept within a median of 3.8 months (IQR 3.3-5.6). Three patients $(2.7 \%)$ restarted treatment with infliximab, golimumab, or adalimumab within a median of 22.7 months (IQR 15.0-35.8) after discontinuing biosimilar etanercept.

The characteristics for retransitioning were explored in the nested case-control study and are presented in Table 2 . Of the 11 patients in the retransitioning cohort (cases) initiating corticosteroids or intensifying immunomodulator treatment, five intensified immunomodulator treatment, five initiated corticosteroid treatment, and one did both. Within the control group, 11 patients intensified immunomodulator treatment, 12 initiated corticosteroid treatment, and one did both. None of the patients initiated immunomodulator treatment.

From the univariate analysis, initiating corticosteroids or intensifying immunomodulator treatment increased the odds of retransitioning by 2.37 (95\% CI 1.03-5.45) compared with patients without changes in corticosteroid or immunomodulator treatment. The frequency of outpatient visits to the rheumatology department in the 60-day period before retransitioning was associated with a significantly increased risk of retransitioning, where the odds increased by 2.06 (95\% CI $1.55-2.74)$ for every additional visit. No other determinants studied were associated with retransitioning. The multivariate analysis revealed that, for each outpatient visit to the rheumatology department, the odds of retransitioning increased by 2.19 (95\% CI 1.60-3.01).

\section{Discussion}

In this study, we investigated the incidence of and reasons for retransitioning to originator etanercept in a cohort of patients with RD who transitioned from originator etanercept to biosimilar etanercept over a median time of 4.4 years. We also explored the determinants for retransitioning. We demonstrated that approximately one in seven patients

Table 2 Association between different patient and treatment characteristics and retransitioning (cases) from biosimilar etanercept to originator etanercept

\begin{tabular}{|c|c|c|c|c|}
\hline Characteristics & Cases $(n=47)$ & Controls $(n=188)$ & OR (univariate model) ${ }^{\mathrm{a}}$ & OR (multivariate model) \\
\hline Age, years & $58.0 \pm 14.5$ & $57.3 \pm 13.9$ & $1.00(0.98-1.03)$ & $1.00(0.97-1.03)$ \\
\hline \multicolumn{5}{|l|}{ Sex } \\
\hline Male & $15(31.9)$ & $90(47.8)$ & Ref & Ref \\
\hline Female & $32(68.1)$ & $98(52.1)$ & $1.95(0.99-3.83)$ & $1.36(0.61-3.05)$ \\
\hline \multicolumn{5}{|l|}{ Biosimilar etanercept dosing interval at index date } \\
\hline 7 days & $35(74.5)$ & $122(64.9)$ & Ref & Ref \\
\hline$>7$ days & $12(25.5)$ & $66(35.1)$ & $0.61(0.29-1.29)$ & $0.63(0.27-1.48)$ \\
\hline \multicolumn{5}{|l|}{ Previous use of other biological } \\
\hline No & $44(93.6)$ & $178(94.7)$ & Ref & Ref \\
\hline Yes & $3(6.3)$ & $10(5.3)$ & $1.00(0.27-3.74)$ & $1.42(0.30-6.67)$ \\
\hline \multicolumn{5}{|l|}{ Duration of originator etanercept treatment } \\
\hline More than 4.3 years & $24(51.1)$ & $93(48.9)$ & Ref & Ref \\
\hline Less than 4.3 years & $23(48.9)$ & $96(51.1)$ & $0.91(0.48-1.75)$ & $0.72(0.32-1.64)$ \\
\hline \multicolumn{5}{|l|}{$\begin{array}{l}\text { Initiating corticosteroids or intensifying immune- } \\
\text { modulator treatment }\end{array}$} \\
\hline No & $36(76.6)$ & $166(88.3)$ & Ref & Ref \\
\hline Yes & $11(23.4)$ & $22(11.7)$ & $2.37(1.03-5.45)$ & $2.12(0.80-5.64)$ \\
\hline \multicolumn{5}{|l|}{ Hospitalization } \\
\hline No & $42(89.4)$ & $168(89.4)$ & Ref & Ref \\
\hline Yes & $5(10.6)$ & $20(10.6)$ & $1.00(0.36-2.76)$ & $0.44(0.12-1.53)$ \\
\hline Number of visits to the rheumatology department & $2.2 \pm 1.7$ & $0.8 \pm 1.1$ & $2.06(1.55-2.74)$ & $2.19(1.60-3.01)$ \\
\hline
\end{tabular}

Data are presented as $n(\%)$, mean \pm standard deviation, or odds ratio (95\% confidence interval) unless otherwise specified. Analysis performed using conditional logistic regression

OR odds ratio, $R e f$ reference

${ }^{a}$ Crude estimates are matched by design on index date (index date between June and August 2016 or index date between September and December 2016)

Significant results are presented in bold 
retransitioned, and most did so within 1 year of transitioning to the biosimilar. The most frequently reported reason for retransitioning was a (perceived) loss of effect after the introduction of the biosimilar. Patients who initiated corticosteroids or intensified immunomodulator treatment, as well as patients who had frequent visits to the rheumatology department, had an increased risk of retransitioning. However, in the multivariate model, only the frequency of visits to the rheumatology department seemed to be associated with retransitioning.

This study demonstrated that $9.4 \%$ of patients had retransitioned to originator etanercept 1 year after the introduction of the biosimilar, which increased to $13.7 \%$ after 4.4 years of follow-up. This finding was in line with those of previous studies in which patients were transitioned in 2016 or 2017 , reporting that $2.7-13.3 \%$ of their patients retransitioned within 6-12 months [7-9].

In the present study, the majority of patients (82.4\%) continued to use biosimilar etanercept 6 months after transitioning from originator etanercept to biosimilar etanercept; this proportion decreased to $69.7 \%$ at 1 year after transitioning. Previous studies on transitioning to biosimilar etanercept in patients with RD, also performed in 2016, reported higher rates of persistence, varying from a 6-month persistence of $90 \%[8,13]$ to a 1-year persistence of $73-83 \%[7,10]$. Political factors such as the availability of the originator, regional/ national policies, and pricing and reimbursement of the originator and the biosimilar are likely to affect the persistent use of the biosimilar and therefore the proportion of patients retransitioning. In our study, originator etanercept was still available, which might (partly) explain the differences found. We acknowledge that retransitioning will not occur if the originator is no longer available or reimbursed. A structured positive framing strategy toward biosimilars has been demonstrated to positively affect patients' opinions regarding biosimilars [14]. Although the letter patients received within our study contained factual information about biosimilars, patients were given the opportunity to discuss their concerns with the rheumatologist and/or rheumatology nurse. However, positive framing direct from the beginning of the implementation strategy might have contributed to higher persistence.

Patients who did not retransition to originator etanercept and who were not persistent with biosimilar etanercept were subdivided into patients who switched to another biological treatment (or to a JAK inhibitor) and patients who discontinued without switching. At 6 months after transitioning, 3.5\% of patients had switched and $7.4 \%$ had discontinued. This was higher than the $3.0 \%$ who discontinued within 6 months after transitioning in the study by Tweehuysen et al. [8]. However, in that study, patients who discontinued because of remission were censored and not counted as discontinued, which explains the lower percentage of discontinued patients found [8].

Three-quarters of the patients who were classified as biosimilar etanercept discontinuers at the end of follow-up restarted treatment with biosimilar etanercept within a few months. This could be because they initially discontinued treatment because they experienced sustained remission [15] but then experienced a flare and restarted treatment [16]. Another explanation for this finding might be that patients who continuously used biosimilar etanercept, but prolonged the dosing interval without informing the outpatient pharmacy, were misclassified as discontinued. To minimize the number of misclassified patients, we used a broad permissible gap of 90 days. However, if the dosing interval more than doubled, patients could still have been misclassified as discontinuers.

Previous studies reported that approximately one-half of patients were not persistent with biosimilar etanercept because of either objective clinical worsening or subjective health complaints $[7,8,10]$, which could be classified as the nocebo effect. The present study demonstrated that the majority of patients retransitioned because of (perceived) loss of effect when treated with the biosimilar. Although retransitioned patients remained treated with the originator etanercept for a median of 2.0 years, which might suggest that patients regained treatment effect, the nocebo effect might have played a role. By contrast, patients in a previous study remained treated with originator etanercept for 0.65 years, but the follow-up was shorter [7]. For the patient, a (perceived) loss of effect, regardless of whether it is classified as a nocebo effect, is a burden and might negatively impact treatment outcome. No patient retransitioned because of the autoinjector used to administer the biosimilar etanercept. This finding is supported by a previous study that examined patient perceptions of the autoinjector of the biosimilar etanercept and the originator etanercept and reported a preference for the biosimilar's autoinjector [17]. Although the biosimilar device was not reported as a reason for retransitioning in the present study, it should be taken into consideration during the introduction and implementation strategy for biosimilars.

In the current study, initiating corticosteroids or intensifying immunomodulator treatment in the 60-day period before the event, as well as the number of visits to the rheumatology department in the 60-day period before the event, increased the odds of retransitioning. However, when other patient- and treatment-related factors were accounted for, only the number of visits to the rheumatology department was associated with retransitioning: each visit in the 60-day period before the event increased the odds of retransitioning by 2.19 . The patients in our study who wished to retransition were extensively contacted by the treating rheumatologist or the rheumatology nurse to discuss their concerns with 
the biosimilar before the decision on retransitioning was made. This might (partly) explain the association. Initiating corticosteroids or intensifying immunomodulator treatment was also not significantly associated with retransitioning in the multivariate (full) model. Although no formal correlation existed between the initiation of corticosteroid treatment or intensification of immunomodulatory treatment and the number of visits to the rheumatology department, some coherence between these determinants is possible. Although transitioning to biosimilar etanercept does not increase disease activity [6], patients might experience a loss of effect, resulting in more complaints, which might be treated by initiating or intensifying corticosteroids or immunomodulator treatment and simultaneously retransitioning to originator etanercept.

For clinical practice, these results highlight that, when patients transition from originator etanercept to the biosimilar, clinicians should anticipate one in seven patients not persisting with biosimilar treatment and retransitioning to the originator. In addition, clinicians should be aware that retransitioning occurs not only in the first few months after transitioning but also later.

This study provides insight into transitioning from originator etanercept to biosimilar in patients with RD using real-world clinical data. As such, it provides a reflection of daily clinical practice and contributes to the limited knowledge regarding retransitioning. We included a heterogeneous patient population in terms of treatment duration and biological treatment history, and we did not restrict inclusion to a certain type of RD or to patients whose diseases were clinically stable.

Moreover, we assessed the incidence of retransitioning over a longer period of time than did previous studies. Our results indicate that retransitioning was not limited to the first months after transitioning, suggesting that studies with a shorter follow-up might have underestimated the incidence of retransitioning.

Several limitations of this study must be addressed. First, the indication for etanercept treatment for the included patients was not known. However, as retransitioning to the originator is not recommended for any RD, we consider it unlikely that this information could have influenced our results and main conclusions. Furthermore, we commenced our data collection in 2016, and experience with biosimilars has increased since then. However, a recent study demonstrated that a gap in healthcare professionals' knowledge about biosimilars still exists [18]. Therefore, studies on patients transitioning from originator to biosimilar are required to increase knowledge about and improve the introduction of future biosimilars in clinical practice. Moreover, by starting data collection in 2016, we were able to follow patients over a longer period of time than did previous studies. Finally, this study was performed in one hospital, which might compromise its generalizability to other settings. However, because our results complement those of similar previous studies, we believe that the information provided by our study is further applicable to other hospitals. As previously discussed, political factors such as the availability of the originator, regional/national policies, and pricing and reimbursement of the originator and the biosimilar are likely to affect the proportion of patients who retransition. Within the present study, the originator was available and reimbursed throughout the study period. This might have an effect on the generalizability of our results.

\section{Conclusion}

The results of this study demonstrate that, despite an extensive implementation strategy, when introducing a biosimilar in clinical care, clinicians should anticipate several patients retransitioning to the originator. The most frequent reason for retransitioning was a perceived loss of effect, followed by adverse events after the introduction of the biosimilar. Patients who visited the outpatient rheumatology department more frequently had an increased risk of retransitioning, which probably reflects patients reporting loss of effect and adverse events, resulting in more visits to the rheumatology department as part of the implementation strategy chosen. The provision of information specifically aimed at the concerns of these patients might prevent them from retransitioning. Therefore, more qualitative studies are required to obtain more detailed information on the underlying reasons for retransitioning from both patients and physicians to improve the introduction of biosimilars in clinical care.

Supplementary Information The online version contains supplementary material available at https://doi.org/10.1007/s40259-021-00501-x.

\section{Declarations}

Funding This research did not receive any specific grants from funding agencies in the public, commercial, or not-for-profit sectors.

Conflict of interest RWM, HG, MLB, SW, TCGE, and TJG have no potential conflicts of interest related to the content of this manuscript.

Ethical approval The protocol of this study was approved by the Institutional Review Board of the Spaarne Gasthuis (reference number 2018.78). Informed consent was waived for this study.

Consent to participate Not applicable.

Consent for publication Not applicable.

Availability of data and materials Research data will not be shared. 
Code availability Not applicable.

Author contributions All authors contributed to the conception or design of the study. RWM, HG, MLB, and TJG contributed to the acquisition of data and data analysis. All authors participated in the interpretation of the data, contributed to the drafting or revision of the manuscript, and read and gave final approval of the submitted manuscript.

Open Access This article is licensed under a Creative Commons Attribution-NonCommercial 4.0 International License, which permits any non-commercial use, sharing, adaptation, distribution and reproduction in any medium or format, as long as you give appropriate credit to the original author(s) and the source, provide a link to the Creative Commons licence, and indicate if changes were made. The images or other third party material in this article are included in the article's Creative Commons licence, unless indicated otherwise in a credit line to the material. If material is not included in the article's Creative Commons licence and your intended use is not permitted by statutory regulation or exceeds the permitted use, you will need to obtain permission directly from the copyright holder. To view a copy of this licence, visit http://creativecommons.org/licenses/by-nc/4.0/.

\section{References}

1. European Medicines Agency. Guideline on similar biological medicinal products; 2014

2. Hofmann HP, et al. Characterization and non-clinical assessment of the proposed etanercept biosimilar GP2015 with originator etanercept $\left(\right.$ Enbrel $\left.^{\circledR}\right)$. Expert Opin Biol Ther. 2016;16(10):1185-95

3. Matucci-Cerinic M, et al. Efficacy, safety and immunogenicity of GP2015, an etanercept biosimilar, compared with the reference etanercept in patients with moderate-to-severe rheumatoid arthritis: 24-week results from the comparative phase III, randomised, double-blind EQUIRA study. RMD Open. 2018;4(2):1-9.

4. Emery P, et al. A phase III randomised, double-blind, parallelgroup study comparing SB4 with etanercept reference product in patients with active rheumatoid arthritis despite methotrexate therapy. Ann Rheum Dis. 2017;76(1):51-7.

5. Matsuno H, Tomomitsu M, Hagino A, Shin S, Lee J, Song YW. Phase III, multicentre, double-blind, randomised, parallel-group study to evaluate the similarities between LBEC0101 and etanercept reference product in terms of efficacy and safety in patients with active rheumatoid arthritis inadequately responding to methotrexate. Ann Rheum Dis. 2018;77(4):488-94.
6. Matucci-Cerinic MJJ et al. Switch between reference etanercept (ETN) and gp2015, an etanercept biosimilar, did not impact efficacy and safety in patients with moderate-to-severe rheumatoid arthritis: 48-week results from the phase 3 equira study. Arthritis Res Ther. 2019; 609-609.

7. Glintborg B, et al. To switch or not to switch: results of a nationwide guideline of mandatory switching from originator to biosimilar etanercept. One-year treatment outcomes in 2061 patients with inflammatory arthritis from the DANBIO registry. Ann Rheum Dis. 2019;78(2):192-200

8. Tweehuysen L, et al. Open-label, non-mandatory transitioning from originator etanercept to biosimilar SB4: six-month results from a controlled cohort study. Arthritis Rheumatol (Hoboken, NJ). 2018;70(9):1408-18.

9. Scherlinger M, Langlois E, Germain V, Schaeverbeke T. Acceptance rate and sociological factors involved in the switch from originator to biosimilar etanercept (SB4). Semin Arthritis Rheum. 2019;48(5):927-32.

10. Müskens WD, Rongen-van Dartel SAA, Teerenstra S, Adang EMM, van Riel PLCM. One-year results after transitioning from etanercept originator to biosimilar in a setting promoting shared decision-making in rheumatology. Rheumatol Adv Pract. 2020;4(2):42.

11. Vlieland ND, Gardarsdottir H, Bouvy ML, Egberts TCG, van den Bemt BJF. The majority of patients do not store their biologic diseasemodifying antirheumatic drugs within the recommended temperature range. Rheumatol (United Kingdom). 2016;55(4):704-9.

12. European Medicines Agency. Product Information Benepali (etanercept); 2019. p. 167-172.

13. Sigurdardottir V, Svärd A. Repeated switches between reference product etanercept and biosimilar do not affect disease activity or retention rate of etanercept over 24 months - a cohort study with historical controls. Jt Bone Spine. 2019;86(4):529-30.

14. Gasteiger $\mathrm{C}$, et al. The effects of message framing on patients' perceptions and willingness to change to a biosimilar in a hypothetical drug switch. Arthritis Care Res (Hoboken). 2020;72(9):1323-30.

15. Gossec L, et al. European League Against Rheumatism (EULAR) recommendations for the management of psoriatic arthritis with pharmacological therapies: 2015 update. Ann Rheum Dis. 2016;75(3):499-510.

16. Moghadam MG, et al. Stopping tumor necrosis factor inhibitor treatment in patients with established rheumatoid arthritis in remission or with stable low disease activity: a pragmatic multicenter, open-label randomized controlled trial. Arthritis Rheumatol. 2016;68(8):1810-7.

17. Thakur K, Biberger A, Handrich A, Rezk MF. Patient perceptions and preferences of two etanercept autoinjectors for rheumatoid arthritis: findings from a Patient Survey in Europe. Rheumatol Ther. 2016;3(2):245-56.

18. Kolbe AR, et al., Physician understanding and willingness to prescribe biosimilars: findings from a US National Survey. BioDrugs. 2021;25(3):363-72. 